Question 38.-Does the principle of free choice and the preservation of the doctor-patient relationship include free choice of patient by doctors working in Health Centres, subject to the limitation of lists? If so, what machinery is proposed to enable a doctor to obtain the transfer of a patient from his list when he feels that the association is no longer beneficial?

Answer--Yes. It is not proposed that doctors in Health Centres should be placed in any worse position in this respect than doctors in public practice outside Health Centres. Details of machinery remain to be discussed.

Question 39.-Will the Minister give an assurance that patients will be allowed a reasonable choice of Health Centre if and when established, and that this choice shall not be unnecessarily limited in the case of patients living near the boundary between two local authority areas?

Answer.-Yes. Every endeavour will be made to secure this.

Question 40.-Why is remuneration by salary considered necessary for practitioners working in Health Centres? In other words, why is competition considered to be incompatible with Health Centre practice?

Question 41.- What is meant by salary or " similar" method of remuneration (p. 32)?

Answer.-This subject is dealt with on pages $31-2$ of the White Paper. The phrase "similar method" is meant to cover any reasonable and practicable method of remuneration which is in accord with the conception of partnership between doctors working together in a Health Centre. A possible example is a small basic salary for all, plus a pooling of the allocatable income of the centre and a sharing among its doctors on an agreed partnership basis. There are other possibilities, no doubt. Suggestions from the profession on this will be welcomed.

Question 42.-Will every woman he entitled to the attendance of a doctor of her choice under the service throughout the ante-natal, natal, and post-natal stages of maternity?

Answer.-This is one of the questions which the Minister particularly wishes to discuss in more detail with the profession before any proposals can be formulated. The main problems will be to determine the extent to which a woman should have a right to require her family doctor under the new service (or any other doctor) to be present at her actual confinement, how far it should rest only with the midwife to call in the doctor if needed. and in that event whether it should normally be the woman's family doctor who is called in.

\section{Medical Officers of Health}

Question 43.-How is it proposed to compensate a medical officer of health for the loss of the intellectual satisfaction to he derived from the varied work for which he is at present responsible when he becomes a subordinate officer in a larger authority or remains the officer of an authority shorn of the functions which have been his main interest for many years?

Answer.- The underlying assumption of this question cannot be accepted. A service of the kind and the scope now proposed will clearly increase, rather than restrict, the field of interest and activity in this branch of professional life.

Question 44.-Are the salaries of medical officers of health to be left to voluntary agreement, while the rates of remuneration of general practitioners and perhaps of specialists are wholly or largely settled by central agreement for the whole country?

Answer.-This is a matter which must be discussed before the new arrangements are settled. Already there is some element of central standardizing of scales for this branch of the profession. and the review of these scales will need to be considered in any case.

A. V. S. Sarma (J. Ind. med. Soc. 1043, 13, 74) records his observations on 25 cases of typhoid fever treated with nicotinic acid, and found that it was devoid of any toxic effect. When used early in the disease it appeared to be particularly beneficial in combating oro-lingual ulceration, excessive salivation, mental symptoms such as confusion and delirium and insomnia, and in reducing length of stay in hospital.

\section{HEAD INJURIES INVOLVING AIR SINUSES}

BY

\section{DONALD McKENZIE, F.R.C.S.}

Major, N.Z.M.C.; Officer in Charge of Base Neurosurgical Unit for Middle East

This paper aims at recording the details and the experience gained of 10 such cases of head injury treated by operation in the neurosurgical unit. Publication of these cases was stimulated by reading a paper on this subject by C. A. Calvert (1942) from the Military Hospital for Head Injuries, and this series lends point to his plea for early operation. Hitherto the tendency has been to adopt an expectant attitude, and, if the rhinorrhoea stops in a few days, as it may easily do, then to do nothing more. In the pre-sulphanilamide days I had the tragic experience of losing such a patient with pneumococcal meningitis in the sixth week, and other cases quoted in the paper referred to show that the danger exists for as many years after injury. In all the cases the anterior fossa was involved, and these observations concern the sinuses of that region and do not include the mastoid sinus.

Anatomy.-The regions which are fraught with the danger of a compound fracture into the subarachnoid space are the posterior wall of the frontal sinus, the ethmoid cells, the cribriform plate itself, and the sphenoidal sinus. The dura may be penetrated by a spicule of fractured bone in any situation, but the floor of the anterior fossa lends itself to an additional method of damaging the dura. The bone in the region specified is extremely thin-even of tissue-paper thinness in places-and the closely attached dura is also very thin. When the base of the skull is fractured either by radiation from the vertex or by general distortion, the dura splits easily in this situation. On several occasions one edge of a dural tear has been removed from a linear fissure which has nipped the dura Hence in the anterior fossa base the thin dura may be penetrated by spicules or split by distortion accompanying a fissured fracture A distinction must be made between trauma of the ethmoid region and that of the cribriform plate. In the former region the fracture must tear the dura, but, in the latter, rupture of the olfactory nerves alone leaves an open pathway to infection. The sphenoidal sinus is least likely to be involved, as most fractures are anterior to this region.

Material.-Four of the 10 cases were battle casualties and 6 were due to blunt injury.

\section{Case Histories}

Case 1.-A cannon-shell splinter penetrated this man's forehead on Nov. 11, 1942, and radiographs showed the track passing via the left frontal sinus, right ethmoids, and right antrum to the right temporal fossa. Rhinorrhoea was noticed a few days after injury, and it persisted irregularly in time and amount. Operation on March 8 (4 months after injury) showed a fistula running through a hole 1 in. by $1 / 4$ in. in the posterior wall of the left frontal sinus and the anterior cribriform plate. Nasal mucosa was visible through the latter. A graft of fascia lata was applied over the opening remaining when the fistulous track was divided. The whole region was powdered with sulphathiazole and the wound closed.

Case 2.-This man had lost both eyeballs on March 27, 1943, from a shell splinter. Cerebrospinal fluid was seen to leak from the left nostril on March 30. With jugular compression later, cerebrospinal fluid ran from the right orbit. $X$-ray report: "Extensive damage to facial bones, left zygoma, left malar, bridge of nose, and fracture of posterior wall of right frontal sinus." Operation on April 12 (16 days after injury) showed a crack of the posterior wall of the right frontal sinus running into the cribriform plate, and C.S.F. gushed out on raising the dura from this crack. A similar vertical crack on the left side ended in a loose piece of bone posteriorly. Dura from both sides of the cribriform plate was stripped back, enabling a large graft of fascia lata to be applied over the whole area after the crista galli had been removed.

Case 3.- This soldier was wounded on March 22, 1943, by a mortar splinter in the forehead, which also destroyed the right eye. He had a mild attack of meningitis on April 16; and on May 7 for the first time a severe leakage of C.S.F. was reported, and $5 \mathrm{c.cm}$. was coltime a severe leakage of C.S.F. was report : A large right frontal bone defect with many fragments in the orbit, but none in-driven." Later films showed a fine linear fracture running vertically down in the midline and crossing to the left side as it approached the cribriform plate. Operation on May 8 (47 days as it approached the cribriform plate. Operation on massing from the right frontal lobe to the open cavity of the frontal sinus. The cribriform plate was intact on both right and left sides. In this case alone the dura was 
opened, the adherent tip of the frontal lobe was amputated and a graft of fascia lata applied inside the dura over the opening, and the dural incision was then closed.

Case 4.-This man was injured by a mine on Dec. 18, 1942 Brownish rhinorrhoea was observed two days later and continued until Feb. 26, 1943, after which there was. never enough C.S.F. to collect, though it was thought to be present. $X$-ray report: "Extensive damage of the left frontal bone, nasal bones, and left anterior fossa. The fracture passes back in the floor of the left anterior fossa." Encephalography on Feb. 11 showed no abnormality Operation on June 17 (6 months after injury) revealed two vertical cracks in the left frontal sinus and one crack in the right frontal sinus running down to a large hole in the posterior wall. A tear in the dura opposite the hole on the right side was sutured with black silk, the crista galli was removed, and a flap of temporal fascia was placed over the area.

Case 5.-This man crashed on a motor-cycle on May 13, 1943 There was no history of C.S.F. leak. Proptosis was present, and vertical dip'opia, due to the depression of the orbital roof and eyeball on the right side to about 1 in. depth. $X$-ray report: "There is a circular fracture involving the right frontal bone down the midline through the frontal sinus (both anterior and posterior walls affected), across the right cribriform plate and orbital roof out to the temporal fossa, and up towards the vertex to join the fracture of the forehead, completing the circle. There are other secondary fractures, and the orbital roof is a pulped mess depressed into the orbit." On July 13 ( 2 months later) operation was undertaken to raise the orbital roof and permit the eyeball to be raised, and also to explore the dura. A large hole was found in the posterior wall of the right frontal sinus. The right orbital roof and supraorbital crest were in pieces and were raised to their normal position. A doubtful minute hole in the dura towards the front of the cribriform plate had a small muscle-graft placed over it. Convalescence was uneventful. The left eyeball began to rise steadily in the orbit, the diplopia lessened, and the ophthalmologist advised that no further operation on the eyeball itself was necessary.

Case 6.-This man was hit on the nose by the breech-block of an anti-aircraft gun on May 28, 1943, fracturing the nasal bone. C.S.F. rhinorrhoea was reported on the third day and lasted for several days. He developed five recurrent attacks of pneumococcal meningitis, each attack being controlled by sulphadiazine or suphathiazole, until eventually he was flown to this unit on July 5. He was in coma in two of these attacks, and the pneumococcus was cultured from the C.S.F. on one occasion. He developed another attack on the day after admission. $X$-ray report: "A fine linear fracture of the posterior wall of the left frontal sinus running back towards the cribriform plate." Operation was undertaken on July 10, this last attack of meningitis settling down in four days. A crack was found on the left side as shown in the $x$-ray film, running down to the midline and across, to end in a small hole on the right side of the cribriform plate, where mucus could be seen in the nose. The dural tear was increased in stripping the dura back off the cribriform plate. The dural tears were sutured with black silk before placing a large graft of fascia over both sides. He was out of bed on the thirteenth day, but developed a mild meningitic attack next day. This cleared up rapidly with sulphadiazine therapy, and there were no further complications. He was quite well when seen in late November.

Case 7.-This man sustained a motor-cycle crash on June 1, 1943, and was operated upon at once in another hospital. Spicules of bone were picked out of the dura, and C.S.F. leak was reported for three days after operation. $X$-ray report: "A long linear gap in the left frontal bone-the frontal sinus opened and several fragments of bone are situated behind the gap, but not necessarily intracranial in position." Operation on July 12 (6 weeks after injury) revealed a hole in the posterior wall of the left frontal sinus. On this side a protruding bone fragment 3 by $1.5 \mathrm{~cm}$. was drawn out from a dural perforation. A crack was found on the right side anterior to the cribriform plate, with adherent dura. On removing this dura C.S.F. ran out. The dural holes were sutured and a graft of temporal fascia applied over the whole area.

Case 8.-This man was injured in an aeroplane crash in 1943. Two lacerations in the forehead had been sutured, but they were infected and both eyes were closed by oedema. A constant discharge of brownish C.S.F. came from the left nostril. Spinal puncture revealed deeply blood-stained fluid. $X$-ray report: "There is a depressed fracture of the right frontal bone into the frontal sinus with comminution of the orbital roof and ethmoids. A fraccure can be traced down through the ethmoids into the maxilla." Operation performed 2 days later revea'ed a loose piece of bone in the posterior wall of the right frontal sinus continuous with the crista galli, which was also fractured. The crista galli and attached sinus wall were removed. There was a small dural tear opposite the crista, and the right orbital plate and ethmoid roof were cracked in many places. A temporal fascia graft was applied over the small dural tear. The supraorbital crest was raised into position and the depressed frontal bone was also elevated. Convalescence was uneventful.

Case 9.-This officer crashed on a motor-cycle on Aug. 13, 1943, and it is probable that the wheel of the oncoming truck went over his head. He reports that C.S.F. leaked from the nose on the second day and continued for several days. There was a right sphenoidal fissure syndrome-i.e., partial loss of the first to sixth cranial nerves. $X$-ray report: "A gross 'block' fracture of the facial bones-the upper limit passing through both fronto-malar areas and roof of the nose to both sides of the cranium, and the lower limit passing through the body of the maxilla, involving both antra." Operation on Aug. 27 (14 days later) explored both sides of the anterior fossa back to the transverse fissured fracture, which was just anterior to both sphenoidal crests. A subsidiary crack into the right ethmoid region was found, with a dural tear in apposition. The optic foramen was laid open and the sphenoidal crest was also removed to leave the optic nerve theca and the sphenoidal fissure exposed. There was a small tear in the optic nerve theca, through which a normal optic nerve could be seen; no blood-clot was present. A graft of fascia lata was applied over the dural tears. Post-operatively he was thought to be developing a frontal lobe abscess, but the appearance of paraesthesia, sensory changes, and muscle weakness in the palate, neck, and limb muscles established a diagnosis of peripheral neuritis. He was seen in December, when the loss of the lower half of the right field of vision was the only remaining sign of the previous paralysis of the first six cranial nerves on the right side. There was still some weakness in the left leg muscles.

Case 10.- This man was injured on Aug. 27, 1943, in a car smash, and there were compound fractures of a forearm and a leg in addition to the severe head injury. C.S.F. rhinorrhoea was free from the right nostril for five days, and irregularly after that. $X$-ray report: "There is a depressed fracture of the fronto-nasal region, with extensions on both sides to the ethmoid plate. The frontal sinuses are involved in the fracture." Operation on Sept. 13 (17 days later) revealed a loose piece of bone in the posterior wall of the left frontal sinus attached to the fractured crista galli. There was a small dural tear on both sides of the crista due to penetration of spicules. The dura was cleared well back on the cribriform plate to enable these holes to be pursestringed with silk, and a graft of fascia lata was superimposed.

\section{Diagnosis}

C.S.F. rhinorrhoea and intracranial aerocele are two indubitable signs that the dura has been torn and that meningitis may be imminent. Aerocele did not occur in any of the 10 cases, but its presence demands investigation and operative repair of the dural injury.

C.S.F. Rhinorrhoea.-In this series the discharge was slight, lasting two days in three cases, three days in two cases, and irregularly for weeks in three cases; in only one case was there a profuse discharge, whereby $5 \mathrm{c.cm}$. of fluid was collected easily on the 46th day. In five cases the onset was delayed several days, presumably until the shrinkage of obstructing blood-clots permitted the fluid to escape. In the one case with profuse rhinorrhoea the discharge did not appear until the 46th day. In the first few days after injury the C.S.F. may be deeply blood-stained from associated cerebral laceration, and any early nasal bleeding should be given careful consideration. Case 6 showed blood-stained fluid, and Cases 4 and 8 a brownish discharge. Localization of the fracture to the same side as the rhinorrhoea has proved to be quite unreliable unless taken in conjunction with the homolateral anosmia and corroborative $x$-ray findings. Cases 2,3 , and 8 showed left rhinorrhoea, but the dural tear was right-sided; and Case 10, with right rhinorrhoea, had bilateral dural tears. Nasal mucus, or tears, may cause doubt when the amount of fluid is scanty; but jugular compression will usually increase the flow, and the presence of sugar will establish the nature of the fluid.

Anosmia.-Though not an outstanding symptom, anosmia is valuable evidence of local injury where it can be established that there has been an alteration in the sensation of smell following the injury. The patient may be too comatose to examine or to give his pretraumatic condition. Also, one or both nostrils may be blocked by swelling. In our cases the man's statement about his previous sense of smell was accepted. Three cases had no sense of smell on either side; two had a slight degree of smell on one or both sides; four had anosmia on the appropriate side; and the one case with no dural tear had normal sensation.

Radiography.-The recognition of a fracture in the floor of the anterior fossa may be an extremely difficult matter. In Case 6 a faint vertical crack was visible in the posterior wall of the sinus, but it could only be surmised that it continued back into the cribriform plate. At operation it was demonstrated that it had crossed the midline and ended in a hole on the opposite side. Case 5 was described as a circular fracture, but it was also surmised that the fracture crossed the orbital and cribriform plates, whereas it could be easily traced up from the temporal fossa across the forehead and down through the frontal sinus. Our procedure was to take stereo- 
scopic antero-posterior and lateral views and then, if necessary, oblique views. Posterior fractures involving the anterior clinoid process may be elucidated by taking an axial view of the optic foramen where the outer boundary is formed by the anterior clinoid process. Cases 9 and 10 were of the type described as a "block" fracture. The central naso-frontal block of bone was detached by two lateral fissures through the ethmoids in the one case and through the temporal and frontal bones in the other.

Spinal Puncture.-I believe that all patients with a head injury should have a spinal puncture. In the case of these anterior fossa fractures, examination of the spinal fluid may give warning meningitis, and also it will check the presence of blood if there has been any doubtful nose-bleeding.

Meningitis.-Meningitis itself may confirm the diagnosis. Case 6 showed no fracture in the original films, but the history of five recurrent attacks of pneumococcal meningitis was accepted as confirmation of a dural tear, and a stereoscopic film revealed a hairline crack in the frontal sinus. Case 3 developed mild meningitisheadache, neck rigidity, pyrexia, and 10 cells per c.mm.- on the 25 th day after injury. This was controlled in a few days by sulphadiazine therapy. On the 46th day he complained of a "running cold in the head," and was observed to have a profuse C.S.F. rhinorrhoea. It may be mentioned, by the way, that, though frank meningitis is diagnosed clinically by delirium, pyrexia, neck rigidity, and spinal fluid containing several thousand cells per c.mm. I cannot remember a case in which an organism could be recovered from the spinal fluid after sulphadiazine therapy had been instituted.

\section{Treatment}

All of these 10 cases were seen some time after their original injury, when they were co-operative and could be investigated fully There should be no urgency in operating on a recently injured head case until the patient has recovered from the cerebral trauma; and, in fact, judged by this series, the diagnosis of a torn dura would not be made for several days or weeks.

Preliminary Treatment.-All cases received prophylactic sulphadiazine-preferably by mouth, but intravenously if necessary. The dosage was $6 \mathrm{~g}$. daily, but if meningitis was present this was increased to $12 \mathrm{~g}$. daily and an initial $6 \mathrm{~g}$. given intravenously. Blood transfusion was rarely necessary, as the operation does not produce shock and was usually completed in two hours.

Anaesthesia.-This was essentially local infiltration of $0.5 \%$ novocain solution after a preliminary hyoscine and morphine injection, which was supplemented by intravenous pentothal drip when necessary. Our procedure was to set up an intravenous saline drip apparatus at the start, and then either blood or pentothal could be rapidly substituted.

Operative Technique.-A coronal incision within the hair line was used in most cases, turning the forehead flap down to expose both supraorbital ridges. In one case only a linear midline scar already present was incised to turn a unilateral flap, but the cosmetic effect of the coronal flap is well worth achieving at the expense of a bigger incision. The bone flap was unilateral or bilateral, dependent on our confidence in the localization of the dural injury. In either case it was hinged on the temporal muscle, and was made with four burr-holes for the unilateral flap and six burr-holes if bilateral. The lower midline burr-hole was placed as low as possible after studying the size of the frontal sinus. Five cases had bilateral bone flaps, and in only one case was there any bleeding from the longitudinal sinus. The dura was stripped up off the orbital plate both medially towards the cribriform plate and posteriorly towards the sphenoidal crest. Any fracture was easily seen, and the dural tear or escape of C.S.F. was looked for on the opposing dural surface. The opposite side was explored similarly. The more difficult case was the fracture of the crista galli or cribriform plate, where, to get at the dural tear adequately, the dura had to be stripped out of the cleft beside the crista galli-a very difficult procedure. The dura could then be stripped back easily to the sella turcica region if necessary. This complete elevation on both sides was necessary in only two cases (Case 6 and Case 10). It allowed the dural holes to be pursestringed with fine silk, and gave a very good exposure, but is best avoided if any smell sensation is still present. Our procedure was then to powder the dura with sulphathiazole, apply a fascial graft over the area like a postage stamp, and seal an edge with diathermy. The crista galli was nibbled away before applying a midline graft, which would be distorted by its presence. Fascia lata was employed in six cases in which a good solid graft was indicated. Temporal muscle fascia was used in three cases, but it is thin and no great area can be covered. A muscle-graft was used in Case 5, where there was a minute and dubious hole. The graft was applied extradurally except in Case 3 , in which the dura was openea widely to permit removal of the adherent anterior lobe. In this case it was much easier to apply the graft intradurally. It is reasonable to suppose that some other cases could be grafted more simply by working intradurally and avoiding a wide'elevation of the dura. But the dural tears are small, and are best located by finding the fracture and then seeing the hole or the escaping C.S.F. in that region. I do not think these small dural tears could be located as well intradurally, and also there is an advantage in avoiding trauma from direct handling of the cerebral lobes.

All bleeding was stopped by diathermy to the dural veins and wax to the bone. It is much more important to have the dural bleeding completely stopped in these low-pressure cases than in a tumour operation, in which the raised pressure will stop any extradural oozing. The whole dura was then powdered and the boneflap and scalp sutured in place in the usual way. Bone chips were employed to fill up the frontal burr-holes.

After-treatment.-No special complication arose. Both eyes were usually closed by frontal oedema for one or two days. Sulphadiazine therapy was continued for five days after operation with the routine dosage of $6 \mathrm{~g}$. daily. These patients had little pain, and some wished to be allowed up on the third day, though this was not permitted until the tenth day. When revisiting the unit on Nov. 12 all patients had been discharged and were understood to be well.

Those patients with complete anosmia did not appear to mind their disability. They could not detect any testing materials in the mouth or nose, though taste was unimpaired. One patient (Case 7) had a constant subjective sensation which he described as a "disinfectant" smell.

\section{Conclusions}

The following are the conclusions arrived at: (1) That the dangers associated with C.S.F. rhinorrhoea need stressing to medical officers. (2) That unless the possibility of dural tearing is kept in mind, the rhinorrhoea, anosmia, and radiological evidence of fracture may be easily overlooked. (3) That every head injury with C.S.F. rhinorrhoea, however slight, should undergo operative repair of the dura rather than risk the vagaries of natural repair. (4) That with the technique used the operation is neither formidable nor dangerous; there were no deaths and no complications in this series.

I wish to thank the War Office for permission to publish this paper, and also my colleagues Lieut.-Col. R. R. Lumsden, E.N.T. Specialist, Major W. H. Milligan, Radiologist, Major J. E. Caughey, Neurologist, and Major H. B. Stallard, Ophthalmogist, for their helpful collaboration in the care of these patients.

\section{REFERENCE}

Calvert, C. A. (1942). J. Laryng. Otol., 57, 499.

\section{USE OF CRUDE PENICILLIUM FILTRATE FOR LOCAL TREATMENT}

\section{J. M. ALSTON, M.B., M.R.C.P.Ed. \\ (Archway Hospital, London)}

The therapeutic value of partially purified penicillin has been established in this country (Florey and Florey, 1943 ; Fleming, 1943 ; Report to War Office, 1943 ; Christie and Garrod, and others, 1944) and in the United States (Keefer et al., 1943), but the use of untreated filtrate of cultures of Penicillium notatum has been very little noticed, although successful results have been obtained with gauze impregnated with the living mould (Hobson and Galloway, 1944).

This communication gives the results of the local use of the untreated filtrate in 24 patients. The strain of $P$. notatum was kindly given by Prof. Fleming, and in growing it for this purpose the medium has been changed from time to time, but has always been a modification of the Czapek-Dox formula. At present it is made according to that of Challinor and MacNaughtan (1943) without the yeast extract which they recommend and with the addition of calcium carbonate. The following constituents are dissolved in a litre of distilled water:

Sodium nitrate $\left(\mathrm{NaNO}_{3}\right)$

Potassium chloride $(\mathrm{KCl})$

Magnesium sulphate $\left(\mathrm{MgSO}_{4}, 7 \mathrm{H}_{2} \mathrm{O}\right)$

Ferrous sulphate $\left(\mathrm{FeSO}_{4}, 7 \mathrm{H}_{2} \mathrm{O}\right)$

Potassium dihydrogen phosphate $\left(\mathrm{KH}_{2} \ddot{\mathrm{PO}}_{4}\right.$

3.0 grammes

Gisodium hydrogen phosphate $\left(\mathrm{Na}_{2} \mathrm{HPO}_{4},{ }_{\text {" }}^{\left.12 \mathrm{H}_{2} \mathrm{O}\right)}\right.$

brand, Corn Products Ltd.).

Distilled water to 1,000 c.cm.

Sterilize in free steam for $1 \frac{1}{2}$ hours in $200-c . c m$. amounts in 1-litre conical flasks. Add $5 \mathrm{c.cm}$. of $10 \%$ solution of sterile calcium carbonate to every $200 \mathrm{c.cm}$. of medium before inoculating. The final $p H$ is about 7 . 